\title{
Enhancing anti-interference ability of molecularly imprinted ratiometric fluorescence sensor via differential strategy demonstrated by the detection of bovine hemoglobin
}

\author{
Jiangru Wang ${ }^{\mathrm{a}}$, Xiaolan Chen ${ }^{\mathrm{a}}$, Xiaoyan Wang ${ }^{\mathrm{b}}$, Qi Kang ${ }^{\mathrm{a}}$, Dazhong Shen ${ }^{\mathrm{a}, *}$, Lingxin Chen $^{\mathrm{c}, *}$ \\ ${ }^{a}$ College of Chemistry, Chemical Engineering and Materials Science, Key Laboratory of Molecular and Nano Probes, Ministry of Education, Shandong Normal University, \\ Jinan, 250014, People's Republic of China \\ ${ }^{\mathrm{b}}$ School of Pharmacy, Binzhou Medical University, Yantai, 264003, People's Republic of China \\ ${ }^{\mathrm{c}}$ Key Laboratory of Coastal Environmental Processes and Ecological Remediation, Yantai Institute of Coastal Zone Research, Chinese Academy of Sciences, Yantai, \\ 264003, People's Republic of China
}

\section{A R T I C L E I N F O}

\section{Keywords:}

Molecular imprinting

Ratiometric fluorescence

Differential measurement

Smartphone

Bovine hemoglobin

\begin{abstract}
A B S T R A C T
The non-specific adsorption of interferents onto the outside surface of molecularly imprinted polymers (MIPs) particles is disadvantageous for selective determination of the template molecules. Based on the fact that the responses of non-imprinted polymers (NIPs) and MIPs particles to non-template molecules are similar, the antiinterference ability of MIPs sensor can be much improved via differential strategy, demonstrated by bovine hemoglobin (BHb) determination. The MIPs for BHb were prepared via surface imprinting and sol-gel process, with graphene quantum dots@ $\mathrm{SiO}_{2} / \mathrm{CdTe}$ quantum dots (GQDs@SiO $2 / \mathrm{CdTe} \mathrm{QDs}$ ) for ratiometric fluorescence measurements. By using a weight factor of 1.283 to compensate the lower specific surface area in NIPs particles, the differential ratiometric fluorescence signals between MIPs and NIPs channels to $2.00 \mu \mathrm{M}$ ovalbumin and phycocyanin are equivalent to $\mathrm{BHb}$ at the concentration of 0.025 and $0.014 \mu \mathrm{M}$, which are only about $11 \%$ and $5.8 \%$ of the interference level in the normal ratiometric fluorescence method, respectively. The differential ratiometric fluorescence measurement was also performed in a smartphone-based device for field analysis of $\mathrm{BHb}$ in urine samples, with the linear range of $0.05-4 \mu \mathrm{M}$ and the detection limit of $13 \mathrm{nM}$.
\end{abstract}

\section{Introduction}

Generally, selective recognition to target analytes is the cornerstone of the chemical or biological sensors. The probes with excellent recognition selectivity, such as enzymes, antibodies, nucleic acids, and aptamers are widely used as the molecular recognition elements in biosensors [1-3]. However, the using of such biological receptors in biosensors faces some of impediments, such as high cost, low stability, and time-consuming protocol for bio-probes immobilization. In the modern sensors, the natural receptors can be replaced by the synthetic materials which mimic the natural antibody-antigen behavior with almost similar specificity and sensitivity without their restrictions [4]. Molecularly imprinted polymers (MIPs) are tailor-made materials with specific recognition sites complementary in shape, size, and functional groups of template molecules, and benefit from advantages such as chemical and physical stability, easy synthesis, reusability and cost-efficient of mass preparation [5-7]. The molecular imprinting technology has been widely used in industrial and environmental applications
[8,9], sample pretreatment [10], and biosensors [11,12]. Compared with MIPs for small molecules, the imprinting of biomacromolecules, like proteins, is still challenging because of large size, flexible conformation, complex structure, and denaturation of template in nonphysiological polymerization conditions $[13,14]$. Among various strategies for biomacromolecules imprinting, surface imprinting based on the sol-gel method has been the most impressive technique owing to its simplicity and remarkable performance $[7,15]$. The sol-gel based MIPs provide highly crosslinked gels, strong abrasion resistance, and having more physical rigidity to the conservation of the delicately imprinted cavities compared with flexible organic polymers [16]. Moreover, the sol-gel reaction occurs in an aqueous media and ambient temperature that prohibits deformation and inactivation of proteins. Meanwhile, the imprinted cavities generated by surface imprinting are located on the surface of polymers, promoting accessibility to binding sites and suppressing the mass transfer resistance.

Ratiometric fluorescence methods are well known for their high sensitivity and self-correction ability [17]. MIPs based ratiometric

\footnotetext{
* Corresponding authors.

E-mail addresses: dzshen@sdnu.edu.cn (D. Shen), lxchen@yic.ac.cn (L. Chen).
} 
fluorescence sensors offer high sensitivity and selectivity [18]. Usually, the fluorescence intensity of the luminophore is measured by a spectrofluorometer. More recently, the combination of novel sensing technologies with smartphones promotes the development of powerful labon smartphone platforms for real-time and on-site detections [19-21]. Through integrating various specifically designed accessories, smartphones with powerful imaging hardware have been successfully engineered as optical quantitation platforms in colorimetry [22,23], fluorometry [24-26], chemiluminescence [27], electrochemiluminescence [28] etc. Compared to traditional analytical instruments, the smartphone-based analytical devices are more portable and cheaper, and have an inherent capacity in data storage, analysis and share [29]. In these up-to-date devices, the images of a group of sample areas can be recorded in one picture, offering high-throughput optical sensing.

Unlike small templates, biomacromolecules have a complex structure with numerous functional groups available for the interaction with functional monomers. Since different regions of a biomacromolecule have various charge and hydrophobicity, some analogous regions may be existed in other templates and could be adsorbed by functional monomers. In other words, biomacromolecules have innumerable heterogeneous binding sites, which could create nonspecific binding through weak interactions. Therefore, despite the devoting great effort for improving the selectivity of MIPs, non-specific adsorption of matrix interferences compounds is inevitable during the sensing process. For a better understanding of this fact, recent synthesized BHb-MIPs are overviewed. BHb is an important protein component of red blood cells and has the function of oxygen transporting, participating in various physiological activities. Thus, it has been frequently used as a model target analyte in protein imprinting technique [30-32]. Newly fabricated BHb-MIPs particles exhibited the imprinting factors (IF) in the range of $2 \sim 5$ [30-35], implying these recognition adsorbents lack enough anti-interferences ability. For example, in the BHb determination using MIPs with IF even as high as 15.2 [36], other proteins such as bovine serum albumin, lysozyme and ovalbumin can change approximately $19 \%$ of fluorescence intensity of BHb at the same concentration, demonstrating considerable interference from these proteins. It is worthy to note that the responses of MIPs and NIPs to these non-template molecules are usually similar [36-42], because the surface properties of MIPs and non-imprinted polymers (NIPs) particles which could inducement of non-specific binding are almost same. Hence, it is possible to eliminate such interferences by using a differential strategy.

In this work, we report an approach to improve the selectivity and reliability of MIPs sensor via differential strategy. As the adsorption behavior of interferents on the MIPs and NIPs is similar, their response can be eliminated greatly in a differential measurement model, especially after correcting the difference in the specific surface area between MIPs and NIPs particles. The applicability of the differential MIPs sensor was demonstrated by using BHb as the model analyte, which was prepared via surface imprinting and sol-gel process (see Fig. 1). To improve the stability of fluorescence signal, the fluorescence intensity ratio of graphene quantum dots (GQDs) in $\mathrm{SiO}_{2}$ core and CdTe quantum dots (QDs) in surface printing layer was measured, suppressing the baseline drift from variations in light intensity and the concentration of MIPs or NIPs particles. The differential ratiometric fluorescence signals were also measured in a smartphone-based fluorescent device, in which the fluorescence images of twin cuvettes filled with MIPs and NIPs particles were captured by the built-in camera. The proposed device offers the advantage of portability, sensitivity and reliability in field analysis.

\section{Experimental}

\subsection{Reagents and instruments}

Acetonitrile, cadmium chloride pentahydrate, citric acid, 3-

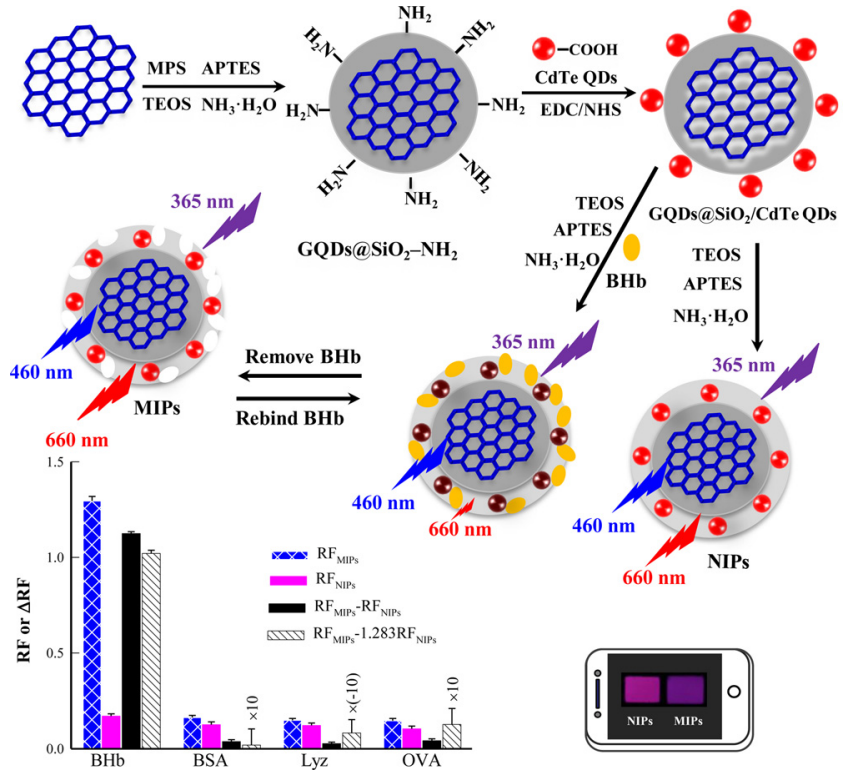

Fig. 1. Schematic illustration for the preparation of fluorescence MIPs sensor toward target $\mathrm{BHb}$ and differential ratiometric determination method.

aminopropyltriethoxysilane (APTES), 3-mercapto propyl trimethoxysilane (MPS), tetraethyl orthosilicate (TEOS), ammonia, 2-N-morpholinoethanesulfonic acid (MES), PBS, 3-ethylcarbodiimide hydrochloride (EDC), and N-hydroxysuccinimide (NHS) were purchased from Shanghai Sinopharm Chemical Reagent Co., Ltd. Sodium borohydride, tellurium powder, thioglycolic acid (TGA), bovine hemoglobin, bovine serum albumin (BSA), lysozyme (Lyz), ovalbumin (OVA), protamine (PA) and phycocyanin (PC) were supplied by Sigma-Aldrich (Shanghai, China). All the reagents were of analytical grade and used directly. Ultrapure water was obtained through ion-exchanger and quartz distiller.

The fluorescence, UV-vis absorption, and infrared absorption spectra were measured on a spectrofluorometer (F-7100, Hitachi), a UV-vis spectrophotometer (UV-1700, Shimadzu), and a Fourier transform infrared (FT-IR) spectrometer (Nicolet iS10, Thermo Fisher Scientific, USA), respectively. The scanning electron microscope (SEM) images were captured on an SEM microscope (Hitachi S-4800 FE). The size distribution measurements were performed on NanoZS90 Zetasizer (Malvern, UK) instrument.

The smartphone-based differential fluorescent device and schematic illustration of differential ratiometric fluorescence MIPs sensor are shown in Fig. S1 in the Electronic Supporting Information (ESI). A $365 \mathrm{~nm}$ LED flashlight light was used as the excitation light source. To obtain better parallel light beam, a collimator made of four laddershaped mirrors was mounted between the light source and the twin cuvettes, where the non-detection areas in the front of the cuvettes were covered by black light-tight film. The fluorescent images were captured by the camera built-in a smartphone (Huawei Honor 7, China). The camera was about $45 \mathrm{~mm}$ apart from the outside wall of the cuvette. The auto-setting function of the camera was turned off and the focus and shooting parameters were adjusted manually. The brightness (unitless, with value ranged $0 \sim 255$ ) of the fluorescence images in the red $(R)$ and blue $(B)$ components in RGB mode was measured by a useredited APP software.

\subsection{Synthesis of GQDs@SiO ${ }_{2} / \mathrm{CdTe} Q D s$}

The preparation of GQDs was carried out according to the previous procedures [43], and the details are described in ESI. The preparation of GQDs@ $\mathrm{SiO}_{2}-\mathrm{NH}_{2}$ is according to the protocol reported previously with slight modification [44], and the details are described in ESI. CdTe 
QDs were synthesized according to our previous work (see ESI) [45].

The composite fluorescent nanomaterials containing CdTe QDs and GQDs@SiO $\mathrm{SH}_{2}-\mathrm{NH}_{2}$ were prepared by EDC/NHS reaction process. In brief, $12 \mathrm{~mL}$ of $10 \mathrm{mg} / \mathrm{mL}$ newly prepared EDC solutions in $0.1 \mathrm{mM}$ MES buffer $\left(\mathrm{pH}=5.5\right.$ ) was added to $10 \mathrm{~mL}$ of GQDs@SiO ${ }_{2}-\mathrm{NH}_{2}$. After stirring for $20 \mathrm{~min}$ at room temperature, $2 \mathrm{~mL}$ of CdTe QDs and $6 \mathrm{~mL}$ of $10 \mathrm{mg} / \mathrm{mL}$ NHS (in $0.1 \mathrm{mM}$ MES buffer, $\mathrm{pH}=5.5$ ) were added into the above solution and dispersed uniformly. The mixture solution was stirred for $12 \mathrm{~h}$ in the dark. The finally obtained CdTe QDs/GQDs@SiO ${ }_{2}$ composites were centrifuged and washed with ultrapure water and then were re-dispersed in $12 \mathrm{~mL} 0.01 \mathrm{M} \mathrm{PBS}$ (pH 7.4) and kept in the dark at $4{ }^{\circ} \mathrm{C}$ for further use.

\subsection{Preparation of $\mathrm{BHb}$ imprinted nanoparticles}

$12 \mathrm{~mL}$ of the as-obtained GQDs@ $\mathrm{SiO}_{2} / \mathrm{CdTe}$ QDs $(1.33 \mathrm{mg} / \mathrm{mL})$ was added to $8 \mathrm{~mL}$ of $0.01 \mathrm{M}$ PBS (pH 7.4). After stirring evenly, $10 \mathrm{mg}$ of $\mathrm{BHb}$ and $20 \mu \mathrm{L}$ of APTES were added into the above solution. After further stirring for $1 \mathrm{~h}, 40 \mu \mathrm{L}$ of ammonia $\left(\mathrm{NH}_{3} \cdot \mathrm{H}_{2} \mathrm{O}\right)$ and $40 \mu \mathrm{L}$ of tetraethyl orthosilicate (TEOS) were added and kept stirring for $2 \mathrm{~h}$ in the dark. The acquired polymers were centrifuged and washed with ethanol/acetonitrile $(8: 2, \mathrm{v} / \mathrm{v})$ to remove $\mathrm{BHb}$, and then were washed with ultrapure water three times. Finally, the obtained MIPs were dispersed in $0.01 \mathrm{M}$ PBS ( $\mathrm{pH} 7.4$ ) and stored in dark at $4{ }^{\circ} \mathrm{C}$. As a comparison, the corresponding non-imprinted polymers (NIPs) were prepared following the similar steps to those of MIPs, except without adding template of $\mathrm{BHb}$. The MIPs of PC were prepared by the same protocol as that of MIPs of BHb by replacing BHb with PC.

\subsection{Analysis of real samples on site}

Three urine samples were randomly collected from healthy bovines from a rural house-hold (Local in Jinan City). The samples were filtered with a $0.22 \mu \mathrm{m}$ microfiltration membrane to remove the particulate suspension and were diluted by 2 -fold volume of $0.01 \mathrm{M}$ PBS ( $\mathrm{pH}$ 7.4). In two plastic centrifugal tubes, $5.00 \mathrm{~mL}$ of the diluted urine sample was added and mixed with $100 \mu \mathrm{L}$ of MIPs or NIPs solution $(2.2 \mathrm{mg} / \mathrm{mL})$, respectively. The mixture of the sample and NIPs was added to the left reference cuvette and mixture of the sample and MIPs to the right sensing cuvette. After an incubation time of $15 \mathrm{~min}$, the fluorescence images of the twin cuvettes were recorded by the smartphone-based portable detection device (Fig. S1). The brightness ratio was calculated by the user-edited APP software to determine the concentration of BHb according to the pre-saved calibration curve. In the recovery experiments, BHb was added in the diluted urine samples at three spiked concentration levels of $0.50,1.00$ and $2.00 \mu \mathrm{M}$. All determinations were performed on site in triplicate.

\section{Results and discussion}

\subsection{Construction and characterization of MIPs}

To perform the ratiometric fluorescence measurement, the blue emissive GQDs was covered by $\mathrm{SiO}_{2}$ shell and used as the reference luminophore, and the sensing luminophore of red emissive CdTe QDs were immobilized on the surface of the $\mathrm{SiO}_{2}$ shell via the EDC/NHS routine (Fig. 1). The imprinting of $\mathrm{BHb}$ on the surface of $\mathrm{GQD} @ \mathrm{SiO}_{2} /$ CdTe QDs was performed via a sol-gel process. It could be seen that MIPs particles present rougher surfaces (Fig. 2A) than the NIPs particles (Fig. 2B), due to the temple removing process. The size distribution of MIPs particles was in the range from 60 to $170 \mathrm{~nm}$ with the average size of $106.7 \mathrm{~nm}$ (Fig. 2C), which was less slightly than that of $122.6 \mathrm{~nm}$ in the NIPs particles (Fig. 2D).

As shown in Fig. S2, the FT-IR spectra of MIPs and NIPs particles were very similar. The characteristic peaks at 1395 and $1589 \mathrm{~cm}^{-1}$ are possibly belonged to the symmetric and antisymmetrical vibrations of the $\mathrm{COO}^{-}$[46]. The absorption band at $1474 \mathrm{~cm}^{-1}$ may be described to the stretching vibration of the propylamine groups $\left(-\mathrm{CH}_{2}-\mathrm{CH}_{2}-\mathrm{CH}_{2}-\mathrm{NH}_{2}\right)$ [47]. These results proved the successful preparation of TGA-QDs and addition of APTES in MIPs and NIPs. The absorption peak at $3419 \mathrm{~cm}^{-1}$ is corresponded to $\mathrm{N}-\mathrm{H}$ stretching vibration, illustrating that amino groups $\left(-\mathrm{NH}_{2}\right)$ were effectively modified onto the surface of MIPs and NIPs particles.

As shown in Fig. S3, the fluorescence excitation peaks of the GQDs and CdTe QDs were around $363 \mathrm{~nm}$ and $376 \mathrm{~nm}$, respectively. The solution of MIPs exhibited two well-separated fluorescence emission peaks around $460 \mathrm{~nm}$ and $660 \mathrm{~nm}$, which were corresponded to those of GQDs and CdTe QDs, respectively. By using quinine sulfate in $0.1 \mathrm{M}$ $\mathrm{H}_{2} \mathrm{SO}_{4}$ (PLQY $=54 \%$ ) as standard reference [48], the photoluminescence quantum yields (PLQY) of GQDs and CdTe QDs in the MIPs were measured to be about $9.0 \%$ and $16.9 \%$, respectively. Under the illumination of $365 \mathrm{~nm}$ UV light, the solution of GQDs and CdTe QDs showed blue and red color, respectively. The pink of solution of MIPs were due to the higher fluorescence intensity of CdTe QDs than GQDs and the higher sensitivity of eye to red than to blue.

\subsection{Performance of the differential ratiometric fluorescence method}

Ratiometric fluorescence methods are well known for their good self-correction ability to the variations in excitation and measurement conditions [17]. In this work, MIPs of GQDs@SiO ${ }_{2} / \mathrm{CdTe}$ QDs were prepared for $\mathrm{BHb}$ determination in a ratiometric fluorescence method. Upon the rebinding to $\mathrm{BHb}$, the red fluorescence of CdTe QDs in the imprinting layer was quenched gradually while the blue fluorescence of GQDs core covered by $\mathrm{SiO}_{2}$ layer was unaffected. Hence, the fluorescence intensity ratio at the wavelength of 460 and $660 \mathrm{~nm}\left(I_{460} / I_{660}\right)$ was measured by a spectrofluorometer and used for the determination of $\mathrm{BHb}$.

As shown in Fig. 3A, with increasing concentration of the MIPs or NIPs particles, the fluorescence intensity at 460 and $660 \mathrm{~nm}$ was increased linearly due to more CdTe QDs and GQDs were available for fluorescence emission. As expected, the intensity ratio of $I_{460} / I_{660}$ is independent of the concentration of MIPs or NIPs particles (Fig. 3B). Because more fluorescence of CdTe QDs in MIPs was quenched by BHb than in NIPs, the value of $I_{460} / I_{660}$ in MIPs is greater than that in NIPs. Importantly, the difference of $I_{460} / I_{660}$ between MIPs and NIPs is also unchanged in the concentration range tested, which offers the convenience for differential ratiometric fluorescence measurements without strictly controlling the concentration of MIPs and NIPs particles. Thus, the differential ratiometric fluorescence signal can obtained from two independent measurements with the same or different concentration of MIPs and NIPs particles. Based on the need of the fluorescence brightness measurement in the smartphone-based device, the concentration of $43 \mu \mathrm{g} / \mathrm{mL}$ was chosen for MIPs and NIPs in the determination of $\mathrm{BHb}$.

\subsection{Condition optimization for $B H b$ determination}

To obtain better analytical performance of the MIPs sensor, $\mathrm{pH}$ and adsorption time were optimized. As can be seen from Fig. S4, in the $\mathrm{pH}$ range from 5.0 to 9.0 , the ratiometric fluorescence signal difference between BHb solution $(2.00 \mu \mathrm{M})$ and blank (indicating by the subscript of 0$),\left(I_{460} / I_{660}\right)-\left(I_{460} / I_{660}\right)_{0}$, was achieved the maximum at $\mathrm{pH} 7.4$. The reason is that protein may be denatured under higher or lower $\mathrm{pH}$. Hence, PBS of $\mathrm{pH} 7.4$ was used for BHb determination.

The response of a MIPs sensor is based on its specific binding to the template molecule. As shown in Fig. S5, the values of $\left(I_{460} / I_{660}\right)-\left(I_{460} /\right.$ $\left.I_{660}\right)_{0}$ were increased with the contact time due to the increasing amount of BHb adsorbed and bound onto MIPs particles, which could quench the fluorescence of CdTe QDs in the imprinting layer. Compared with MIPs, the fluorescence response of NIPs was smaller because less $\mathrm{BHb}$ molecules were adsorbed onto NIPs particles due to the relatively 

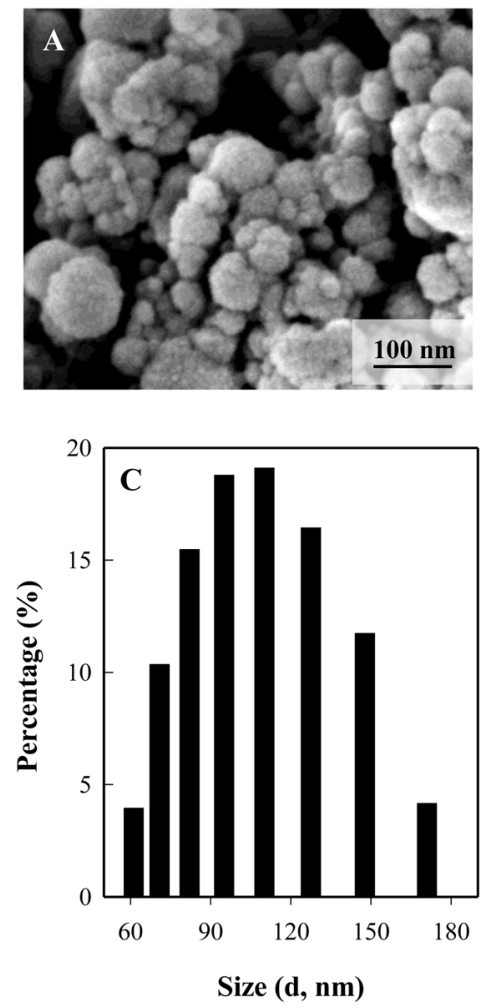
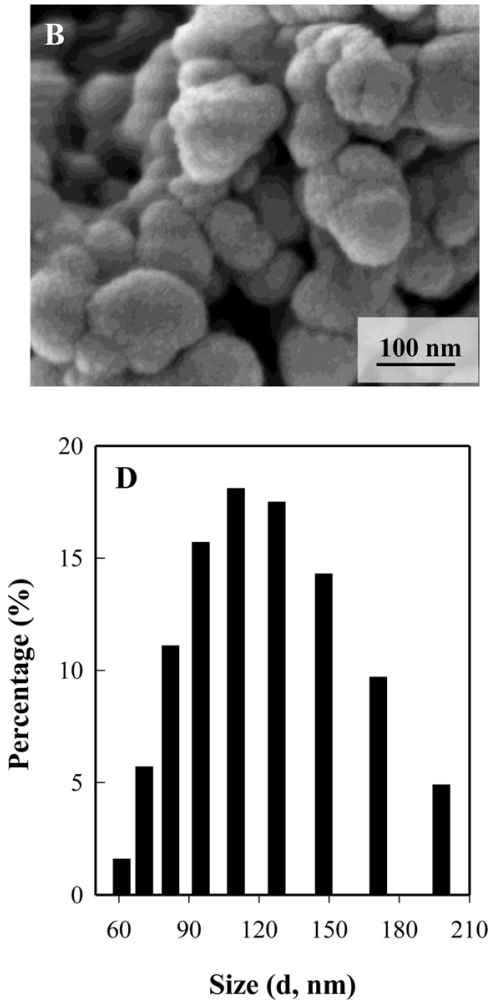

Fig. 2. SEM images and size distribution histograms of the (A, C) MIPs and (B, D) NIPs particles.

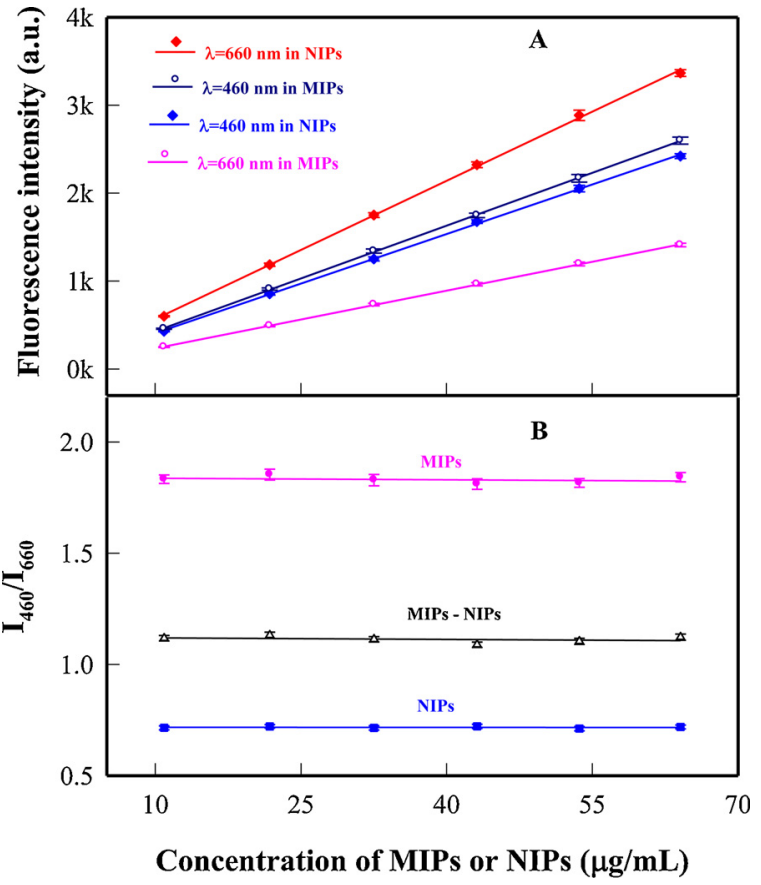

Fig. 3. Influence of the concentration of MIPs or NIPs particles on (A) the fluorescence intensity of GQDs and CdTe QDs and (B) ratiometric fluorescence signals in the MIPs or NIPs in contact with $4.00 \mu \mathrm{M}$ BHb solution.

smooth surface without imprinted cavities. Under the experimental conditions, the response was increased rapidly in the initial $8 \mathrm{~min}$ and then approached gradually to the stable level. The stable response in NIPs was obtained at shorter contact time due to the relatively smooth surface of NIPs particles. The differential ratiometric signal was nearly stable after a contact time of $15 \mathrm{~min}$, which was used as the incubation time for BHb determination.

\subsection{Sensitivity and selectivity of MIPs sensor for BHb determination}

As can be seen in Fig. 4A, with increasing concentration of $\mathrm{BHb}$, the fluorescence intensity of CdTe QDs in MIPs was reduced gradually while fluorescence intensity of GQDs was near the same due to the protection of the $\mathrm{SiO}_{2}$ coating layer. By using the ratiometric fluorescence intensity in MIPs, $R F_{\text {MIPs }}=\left[\left(I_{460} / I_{660}\right) /\left(I_{460} / I_{660}\right)_{0}\right]_{\text {MIPs }}-1$, as the signal indicator, the calibration curve in the MIPs sensor showed acceptable linearity in the concentration of $0.03-4 \mu \mathrm{M}$ (curve a in Fig. 4C). Based on the $3 \sigma$ rule ( $\sigma$ is the standard deviation in blank), the LOD of the ratiometric fluorescence MIPs method was $11 \mathrm{nM}$, offering acceptable sensitivity in compared with other method for BHb determination (see Table 1).

As depicted in Fig. 4B, the fluorescence intensity of CdTe QDs in NIPs was reduced slightly with increasing $\mathrm{BHb}$ concentration. The reason is that part of BHb can be adsorbed on the outer surface of NIPs particles to quench the fluorescence of CdTe QDs nearby. The ratiometric fluorescence intensity in NIPs, $R F_{\text {NIPs }}=\left[\left(I_{460} / I_{660}\right) /\left(I_{460} /\right.\right.$ $\left.\left.I_{660}\right)_{0}\right]_{\mathrm{NIPs}}-1$, was also increased with increasing concentration of BHb (curve b in Fig. 4C). According to the slope of calibration curve in MIPs and NIPs channels, the IF value was estimated to be 7.36, indicating the existence of numerous imprinted cavities in MIPs particles shell to adsorb BHb molecules. As listed in Table 1, the IF values for BHb in other MIPs protocols are in the range of $1.82 \sim 15.2$. The IF of 7.36 in MIPs of GQDs@SiO ${ }_{2} / \mathrm{CdTe}$ QDs was relatively high, offering the acceptable selectivity for the determination of BHb.

As an artificial antibody, MIPs are expected to form a highly specific three-dimensional recognition cavity to recognize template molecules in shape, size and function [5-7]. However, the outer surface of MIPs particles is exposed freely to various molecules in solution. As a result, the non-specific adsorption of other species on the surface of MIPs particles may also change of the signal intensity, causing systematic errors in the determination of target analytes. For example, the presence of other proteins can also result in the decrease of the fluorescence intensity of CdTe QDs in MIPs (Fig. 5). According to the 

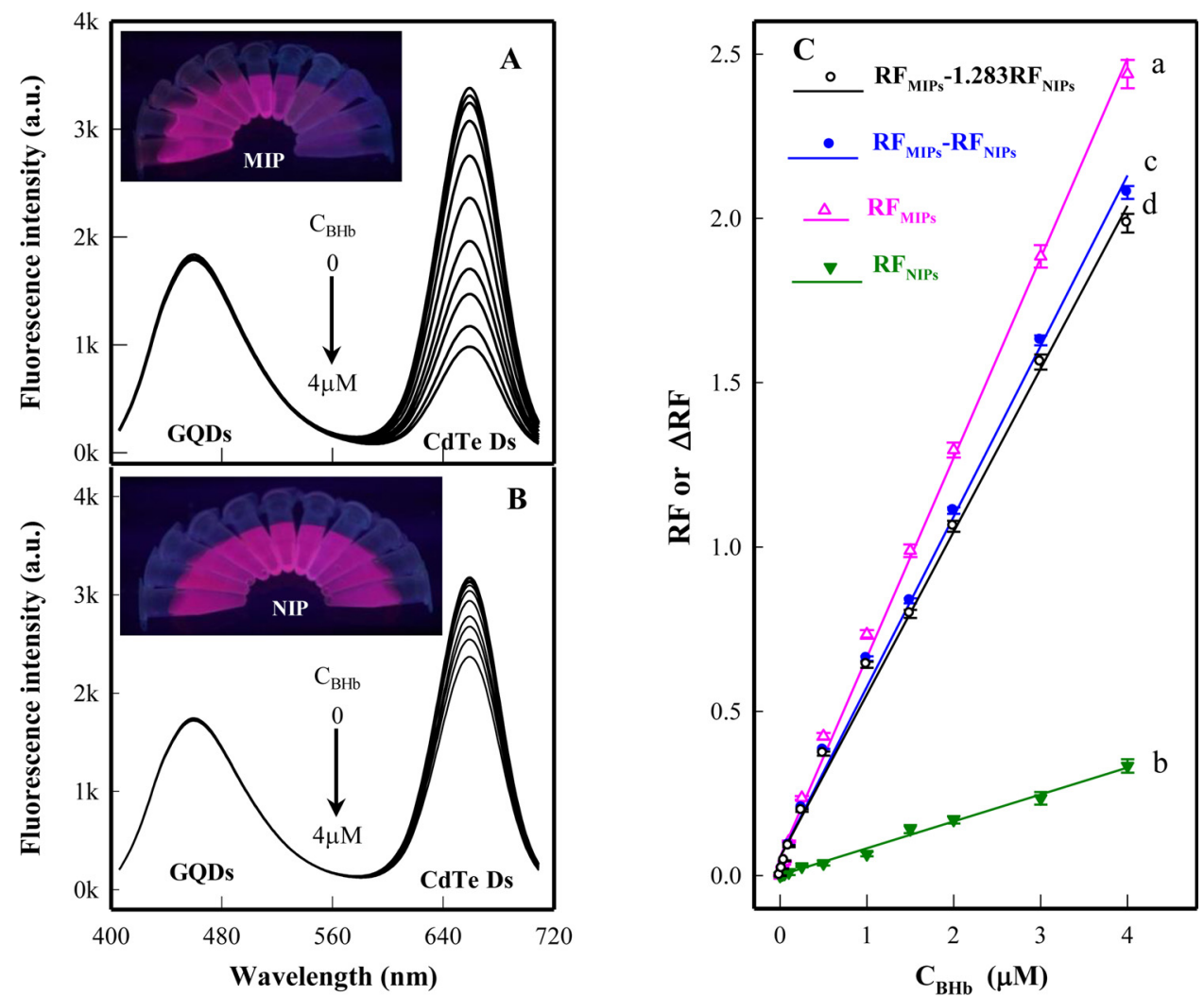

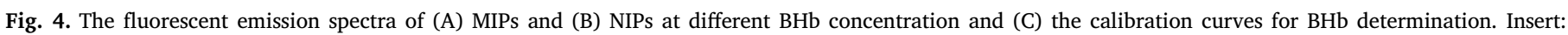
fluorescence images of MIPs + BHb and NIPs + BHb under illumination of UV lamp of $365 \mathrm{~nm}$.

calibration curve in normal ratiometric fluorescence model (curve a in Fig. 4C), $2.00 \mu \mathrm{M}$ of BSA, Lyz, OVA, PA, and PC were equivalent to BHb at the concentration of $0.251,0.228,0.225,0.201$, and $0.236 \mu \mathrm{M}$, respectively, implying minor determination errors for BHb determination. It can be seen that the non-specific adsorption of other species on the outer surface of MIPs is one of the errors source in the determination of template molecule. Interestingly, except BHb, the responses of MIPs and NIPs particles to other proteins were very similar. Hence, the anti-interference ability of the MIPs method is expected to improve greatly in a differential ratiometric mode, which uses the response of NIPs channel as the reference. In this work, the differential ratiometric fluorescence signal $(\triangle R F)$ was calculated by eq. (1).

$\Delta R F=R F_{\mathrm{MIPs}}-R F_{\mathrm{NIPs}}=\left[\frac{I_{460}\left(I_{660}\right)_{0}}{I_{660}\left(I_{460}\right)_{0}}\right]_{\mathrm{MIPs}}-\left[\frac{I_{460}\left(I_{660}\right)_{0}}{I_{660}\left(I_{460}\right)_{0}}\right]_{\mathrm{NIPs}}$

Under the used conditions, the values of $\Delta R F$ were also in good linear correlation with the concentration of BHb (curve c in Fig. 4C). Significantly, the $\triangle R F$ values of the five non-target proteins $(2.00 \mu \mathrm{M})$ were equivalent only to $\mathrm{BHb}$ at the concentration range of
$0.044-0.074 \mu \mathrm{M}$. The interference from the five proteins in $\Delta R F$ signal was only $20 \% \sim 32 \%$ of that in normal ratiometric fluorescence method.

It should be noted that the responses of the NIPs particles is due to the non-specific adsorption of the proteins on their surface. As the surface of NIPs particles is smoother than that of MIPs particles, the smaller specific surface area in NIPs particles is speculated as the reason for the less response of NIPs to non-target proteins compared with MIPs particles. Hence, a weight factor $(\alpha)$ is used to compensate the lower specific surface area in NIPs particles. In this work, a modified differential ratiometric fluorescence method described in eq. (2) was used to improve further the anti-interference ability of the MIPs sensor.

$\Delta R F^{\prime}=R F_{\mathrm{MIPs}}-\alpha R F_{\mathrm{NIPs}}=\left[\frac{I_{460}\left(I_{660}\right)_{0}}{I_{660}\left(I_{460}\right)_{0}}\right]_{\mathrm{MIPs}}-\alpha\left[\frac{I_{460}\left(I_{660}\right)_{0}}{I_{660}\left(I_{460}\right)_{0}}\right]_{\mathrm{NIPs}}$

By using $2.00 \mu \mathrm{M}$ BSA solution as the balance point $\left(\Delta R F^{\prime}=0\right)$, $\alpha=1.283$ was estimated to compensate the difference in specific surface area between MIPs and NIPs particles. It can be seen that the interference of the non-target proteins was suppressed further in the

Table 1

Performance comparison with other MIPs fluorescent methods for BHb determination.

\begin{tabular}{|c|c|c|c|c|c|}
\hline Sensors & Emission mode & $\begin{array}{l}\text { Linear range } \\
\mu \mathrm{M}\end{array}$ & $\begin{array}{l}\text { LOD } \\
\mu \mathrm{M}\end{array}$ & IF & Ref. \\
\hline M-CDs@MIPs & single & $0.05-16$ & 0.017 & 4.1 & {$[30]$} \\
\hline Si-NP/CdTe/MIPs & single & $0.02-2.1$ & 0.0094 & 3.8 & [50] \\
\hline UCNPs/MOFs/MIPs & single & $1.6-9.3$ & 0.96 & 1.82 & [51] \\
\hline CdTe@MIPs+CdTe@SiO 2 & dual & $0.05-3$ & 0.0096 & 6.8 & [47] \\
\hline (Mn)ZnS QDs/MIPs & dual & $0.1-5$ & 0.038 & 3.1 & [52] \\
\hline CdTe/M-R-MIPs & dual & $0.02-2$ & 0.0063 & 10.8 & [53] \\
\hline R-MIPs + G-MIPs + B-MIPs & ternary & $0.025-3$ & 0.0078 & 15.2 & [36] \\
\hline \multirow[t]{2}{*}{ GQDs@SiO ${ }_{2}$-CdTe QD, MIPs - NIPs } & ratiometric & $0.03-4$ & 0.011 & 7.36 & This work \\
\hline & differential ratiometric & $0.03-4$ & 0.0083 & & \\
\hline
\end{tabular}




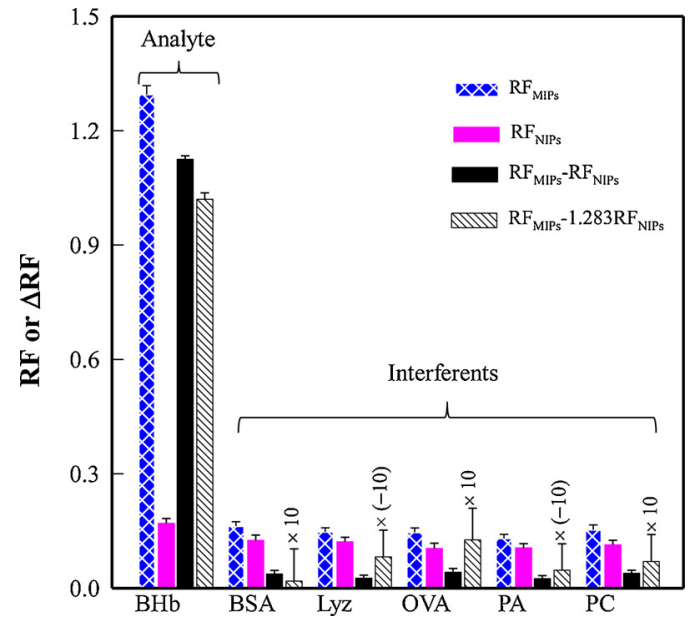

Fig. 5. The ratiometric fluorescence responses of MIPs and NIPs to different proteins $(2.00 \mu \mathrm{M})$ and the differential ratiometric fluorescence response between MIPs and NIPs channels.

modified differential method. Under the experimental used conditions, the presence of $2.00 \mu \mathrm{M}$ Lyz, OVA, PA, and PC resulted in the errors of $-0.016,0.025,-0.0092$, and $0.014 \mu \mathrm{M}$ in $\mathrm{BHb}$ determination, respectively, which was only about $-7.1 \% \sim 11 \%$ of the interference levels in the normal ratiometric fluorescence method. Among the six proteins test, PC solution emits weak fluorescence in the visible spectral region of $600-780 \mathrm{~nm}$ peak at $672 \mathrm{~nm}$ with the excitation wavelength of $532 \mathrm{~nm}$ [49]. But the fluorescence from low concentration PC solution is much weaker than that from CdTe QDs in MIPs and NIPs. Moreover, most of the weak fluorescence from PC solution can be offset in the differential strategy. The emission fluorescence spectra of $\mathrm{BHb}$ and other non-template proteins tested are in the ultraviolet spectral region, which were not excited and detected under the measurement conditions used in this work. Hence, the possible interference from the weak fluorescence in solution except MIPs and NIPs is also much suppressed by the proposed differential method.

In addition, although the slope of the calibration curve in the modified differential model (curve d in Fig. 4C) was only $81.5 \%$ of that in the normal ratiometric one (curve a in Fig. 4C), the error bars in the former were also less than those in later. As a result, the LOD in the modified differential ratiometric method was lowered to $0.0083 \mu \mathrm{M}$, which was even lower than that of $0.011 \mu \mathrm{M}$ in the normal ratiometric fluorescence model.

The reusability and stability are the important MIPs sensor performance metrics [41]. After a group of determinations, the MIPs and NIPs particles were collected separately by centrifugation, and washed with ethanol/acetonitrile $(8: 2, \mathrm{v} / \mathrm{v})$ and ultrapure water to regenerate them for reuse. As shown in Fig. S6A, the fluorescence intensity from CdTe QDs in MIPs and NIPs were decayed slightly with increasing recycles. But most of the recognition ability of MIPs to BHb was remained in the regenerated MIPs particles, especially in the ratiometric fluorescence model (Fig. S6B). For example, after five recycles, the value of $\left(\mathrm{I}_{460} /\right.$ $\left.\mathrm{I}_{660}\right) /\left(\mathrm{I}_{460} / \mathrm{I}_{660}\right)_{0}$ of the regenerated MIPs particles is about $85 \%$ of the initial value using fresh MIPs particles. Because the ratiometric fluorescence signal in NIPs is less obviously than that MIPs, the differential strategy is shown low efficiency to correct the signal decay in the regenerated MIPs. After storing in dark for 3 months, the ratiometric fluorescence signal in MIPs is still $93.1 \%$ of the original value. These results demonstrated that the reusability and stability of the prepared differential ratiometric fluorescence MIP sensor is satisfactory.

It is worth mentioning that the proposed differential approach may be applied to other MIPs systems. As reported previously, the responses of MIPs and NIPs particles to the non-target species are also similar even very close in MIPs methods for the determination of 2,4- dichlorophenoxyacetic acid [54], PC [55], dopamine [56], glycoprotein ovalbumin [57], pyrethroid metabolite [58], hepatitis viruses [41], Japanese encephalitis virus [42], omethoate [59], and Brilliant Blue [60]. If the differential approach had been used, the anti-interference ability of these MIPs method would have been improved greatly.

To address above assumption, the MIPs for PC were prepared by the same protocol as that used in MIPs for BHb. As shown in Fig. S7, the MIPs exhibited obviously enhanced $R F$ signal to PC in compared with NIPs. The IF for PC was estimated to be about 5.63. As the $R F$ signals of MIPs and NIPs to BSA, Lyz, OVA, PA, and BHb are similar, $R F$ signal differences between MIPs of PC and NIPs for the five non-temple proteins is much less than the normal $R F$ signals, especially in the modified differential method with $\alpha=1.19$. Under the experimental conditions used, the presence of $2.00 \mu \mathrm{M}$ of BSA, Lyz, OVA, PA, or BHb were caused the errors in PC determination by $0.334,0.310,0.251,0.288$ or $0.431 \mu \mathrm{M}$ in the normal RF method, $0.0672,0.0691,0.0426,0.0663$ or $0.0650 \mu \mathrm{M}$ in the differential RF method, and 0.0026, 0.111, -0.0773 , 0.0135 or $-0.0211 \mu \mathrm{M}$ in the modified differential RF method, respectively. These results demonstrated again the good efficiency of the modified differential strategy in enhancing the anti-interference ability in the MIPs method.

\subsection{Performance of smartphone-based differential ratiometric fluorescence device}

As can be seen from the insert in Fig. 4A, the color of the MIPs solution was changed from rose-red to blue, which could be applied to the visual determination of BHb with the LOD about $0.5 \mu \mathrm{M}$. To improve the sensitivity and reliability in visual determination, the fluorescence images of the MIPs solution can be recorded by a camera in a smartphone and the brightness of the three primary color of red, green and blue can be measured for quantification. In this work, the differential ratiometric measurements were also performed in a smartphonebased fluorescence device equipped with twin cuvettes (Fig. S1). According to the fluorescence excitation spectra in Fig. S3, a $365 \mathrm{~nm}$ LED light source was used to excite GQDs and CdTe QDs in the MIPs and NIPs channels. The wavelength ranges of the RGB components are corresponded to $560-700 \mathrm{~nm}, 500-600 \mathrm{~nm}$, and $360-510 \mathrm{~nm}$ [61], respectively. It could be seen that the fluorescence emission spectra of GQDs and CdTe QDs were mainly distributed on the blue $(B)$ and red $(R)$ components, respectively. The fluorescence intensity of the two peaks were recorded in the same image and indicated by the brightness values in the blue and red components, which was measured by a useredited APP image analysis software.

As a cheap light source, the intensity of the LED light is related to the available capacity of the rechargeable battery. To test the self-correction ability of the brightness ratio, the light source intensity was adjusted artificially by changing the working voltage of a DC regulated power supply, and measured by the illustration sensor of the smartphone. As shown in Fig. 6A, with increasing excitation light intensity, the values of $R$ and $B$ in the MIPs and NIPs channels were increased near-linearly. However, the influence of the variation in light source intensity on the brightness ratio was much less than brightness (Fig. 6B). For example, with the excitation light intensity increased from 1.41 to $2.81 \mathrm{klx}$ (lx is the unit of luminance), the $R$ values in the MIPs and NIPs channels were increased by $46.1 \%$ and $32.2 \%$, respectively. But values of $B / R$ in MIPs and NIPs channels were $1.0604 \pm 0.0066$ and $0.8568 \pm 0.0171$ (mean \pm standard deviation), with the relative standard deviation (RSD) of $0.62 \%$ and $2.0 \%$, respectively. Noted that the RSD in the MIPs channel was obviously less than that in the NIPs channel, which was due to the larger difference between $R$ and $B$ values in the later. As reported previously [62], the brightness of an image is in a non-linear correlation with the fluorescence intensity. Hence, the corrected brightness $\left(H_{\mathrm{R}}\right.$ and $\left.H_{\mathrm{B}}\right)$ in eq. (3) and (4) are used as the indicator of the fluorescence intensity of CdTe QDs and GQDs. 


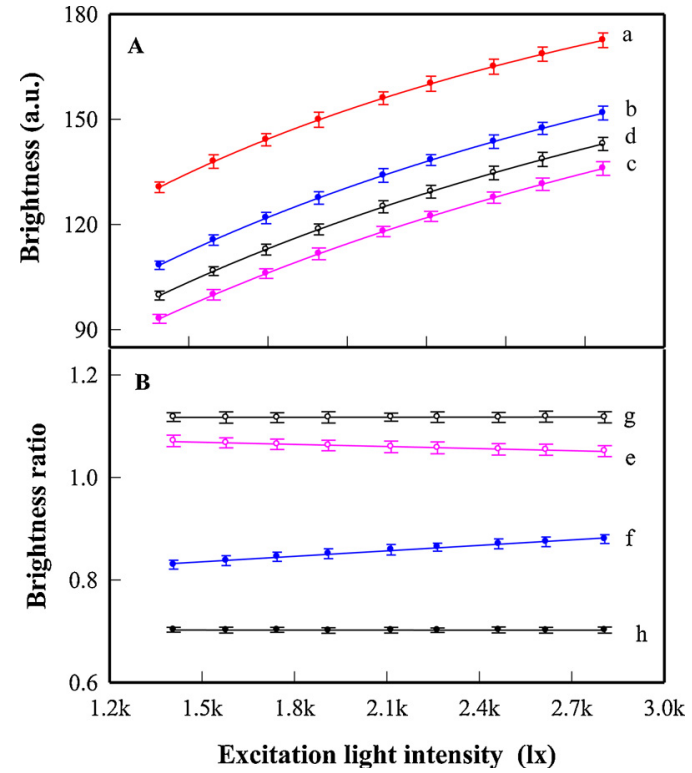

Fig. 6. Influence of excitation light intensity on the (A) brightness and (B) brightness ratio in the MIPs and NIPs channels. (a) and (b) R and B in NIPs channel images, (c) and (d) R and B in MIPs channel images, (e) and (f) B/R in the MIPs and NIPs channels, (g) and (h) HB/HR in the MIPs and NIPs channels. The concentration of $\mathrm{BHb}$ was $1.00 \mu \mathrm{M}$.

$H_{R}=\frac{R-R_{0}}{255-R}$

$H_{B}=\frac{B-B_{0}}{255-B}$

where $R_{0}$ and $B_{0}$ are the background values of the red and blue components in RGB mode, respectively. The ratio of $H_{\mathrm{R}}$ to $H_{\mathrm{B}}$ is given by eq. (5).

$\frac{H_{R}}{H_{B}}=\frac{\left(R-R_{0}\right)(255-B)}{\left(B-B_{0}\right)(255-R)}$

As shown in Fig. $6 \mathrm{~B}$, the corrected brightness ratio, $H_{\mathrm{B}} / H_{\mathrm{R}}$, was remained nearly constant in the excitation light intensity tested range. The values of $H_{B} / H_{R}$ in MIPs and NIPs channels were $1.1174 \pm 0.0006$ and $0.7021 \pm 0.0005$, demonstrating the good self-correction of the ratiometric fluorescent signal in the smartphone-based device with simultaneously recording the fluorescence brightness of GQDs and CdTe QDs in MIPs and NIPs channels. In the twin cuvettes, the excitation light intensity in right channel is less slightly than that in the left channel, which is caused by the absorption and scatting of the solution in left channel and the well of the cuvette. But the influence of excitation light intensity loss on the fluorescence brightness of GQDs and CdTe QDs in the right channel is the same and can be corrected by the ratiometric signal. Consequently, the order of MIPs and NIPs channels in the cuvette can be exchanged if needed and the calibration curve can be saved for subsequent determinations on site. On the other hand, the light source can be powered off except capturing the images, prolonging the service time of the rechargeable battery.

In this work, a $365 \mathrm{~nm}$ LED light source was used to excite GQDs and CdTe QDs in the MIPs and NIPs channels. As can be seen from Fig. S3, the fluorescence emission spectrum of GQDs was overlapped with the fluorescence excitation spectrum of CdTe QDs. As a result, part of the fluorescence of CdTe QDs in the MIPs and NIPs particles is excited by the blue fluorescence from GQDs. But the fluorescence intensity of GQDs is much weaker than the intensity of $365 \mathrm{~nm}$ excitation light source. The reason is that only a small fraction of the excitation light (usually $<5 \%$ ) was absorbed by GQDs in MIPs solutions at low concentration $(43 \mu \mathrm{g} / \mathrm{mL})$ while the PLQY of GQDs in the MIPs was about
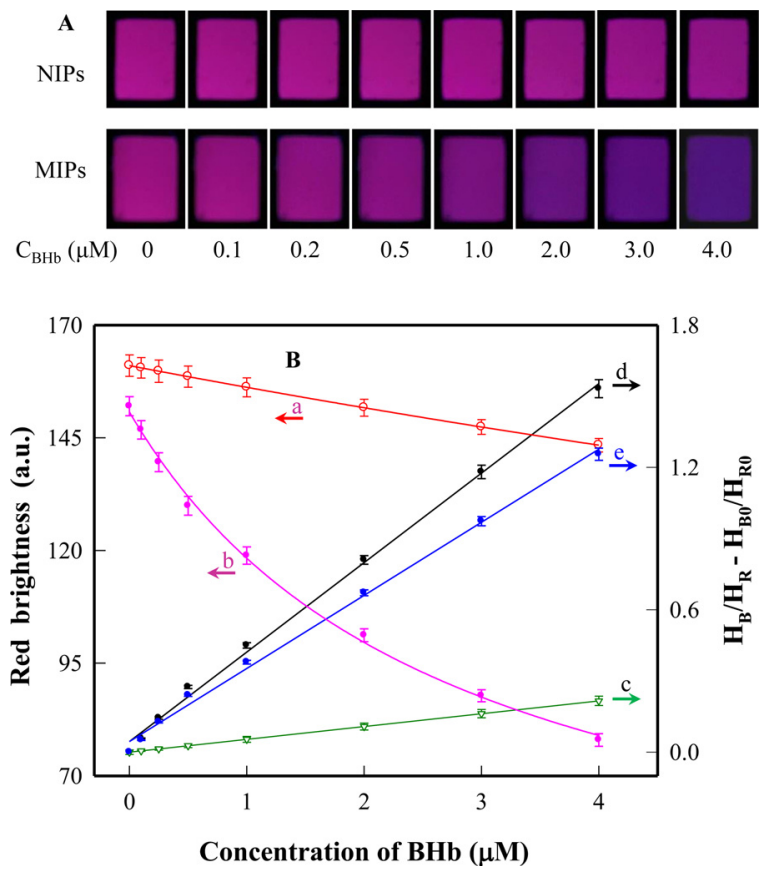

Fig. 7. (A) The fluorescent images of the twin cuvettes at different BHb concentration and (B) the calibration curves for BHb determination in smartphonebased fluorescence method. (a) and (b) R values in the images of NIPs and MIPs solutions, (c) and (d) ratiometric fluorescence calibration curves of NIPs and MIPs to $\mathrm{BHb}$, (e) modified differential ratiometric fluorescence response $(\alpha=1.283)$ to $\mathrm{BHb}$.

$9.0 \%$. Thus, fluorescence intensity of CdTe QDs excited by the blue fluorescence of GQDs is insignificant, especially by the blue fluorescence from the nearby channel in the twin cuvettes due to the limit of emission direction of fluorescence. In addition, the fluorescence intensity of GQDs is stable as they were isolated from the solution by $\mathrm{SiO}_{2}$ coating layer. Hence, the influence of the blue fluorescence of GQDs on the fluorescence intensity of CdTe QDs in the nearby channel is ignorable.

\subsection{Application of field analysis of $B H b$ in real samples}

To demonstrate the applicability of the portable device and the differential MIPs method, BHb in bovine urine samples was determined on site. As shown in Fig. 7A, compared with the NIPs channel, the red color of the fluorescence images in the MIPs channel was decayed more obviously with increasing $\mathrm{BHb}$ concentration, indicated by the decrease in $R$ values in Fig. 7B. By using the correction brightness ratio of $\mathrm{H}_{\mathrm{B}} / \mathrm{H}_{\mathrm{R}}$ as the signal indicator, the calibration curve showed acceptable linearity in the concentration of $0.05-4 \mu \mathrm{M}\left(r^{2}=0.9983\right)$, with the LOD of $0.013 \mu \mathrm{M}$. It can be seen that the simple smartphone based portable fluorescence device has the near same sensitivity as the spectrofluorometer. In this work, the differential ratiometric fluorescence signals between the MIPs and NIPs channels in the smartphone based device is calculated by eq. (6).

$$
\begin{aligned}
\left(\frac{H_{\mathrm{B}}}{H_{\mathrm{R}}}\right)_{\mathrm{MIPs}}-\left(\frac{H_{\mathrm{B}}}{H_{\mathrm{R}}}\right)_{\mathrm{NIPs}}= & \frac{\left(B_{\mathrm{MIPs}}-B_{\mathrm{MIPs} 0}\right)\left(255-R_{\mathrm{MIPs}}\right)}{\left(R_{\mathrm{MIPs}}-R_{\mathrm{MIPs} 0}\right)\left(255-B_{\mathrm{MIPs}}\right)} \\
& -\frac{\left(B_{\mathrm{NIPs}}-B_{\mathrm{NIPs} 0}\right)\left(255-R_{\mathrm{NIPs}}\right)}{\left(R_{\mathrm{NIPs}}-R_{\mathrm{NIP} 0}\right)\left(255-B_{\mathrm{NIPs}}\right)}
\end{aligned}
$$

As discussed above, the differential ratiometric fluorescence signal was insensitive to the variations in the measurement conditions such as light source intensity and the concentration of MIPs and NIPs, which is advantage for field analysis of the portable device.

As listed in Table 2, the concentration of BHb in two bovine urine 
Table 2

Recoveries for BHb determination in the 3-fold diluted bovine urine samples by the differential ratiometric MIPs sensor.

\begin{tabular}{llll}
\hline Bovine urine & Spiked $(\mu \mathrm{M})$ & Found $(\mu \mathrm{M})^{*}$ & Recovery (\%) \\
\hline Sample 1 & 0 & $0.081 \pm 0.011$ & not available \\
& 0.500 & $0.566 \pm 0.017$ & 97.0 \\
& 1.000 & $1.110 \pm 0.026$ & 102.9 \\
Sample 2 & 2.000 & $2.002 \pm 0.037$ & 96.0 \\
& 0 & $0.103 \pm 0.012$ & not available \\
& 0.500 & $0.122 \pm 0.016$ & 103.8 \\
Sample 3 & 1.000 & $1.065 \pm 0.023$ & 96.2 \\
& 2.000 & $2.139 \pm 0.051$ & 101.8 \\
& 0 & not detectable & not available \\
& 0.500 & $0.517 \pm 0.015$ & 103.4 \\
& 1.000 & $0.987 \pm 0.021$ & 98.7 \\
& 2.000 & $2.026 \pm 0.053$ & 101.3 \\
\hline
\end{tabular}

* mean \pm standard deviation $(\mathrm{n}=3)$.

samples was detectable by the proposed smartphone based differential ratiometric fluorescence method. In addition, recoveries were determined by spiking $\mathrm{BHb}$ to the bovine urine samples at three concentration levels of $0.500,1.00$ and $2.00 \mu \mathrm{M}$. The recoveries were in the range from $96 \%$ to $104 \%$, indicating that the proposed device and MIPs method were successfully applied in the detection of BHb in biological samples on site.

\section{Conclusions}

In summary, the differential response signal between the MIPs and NIPs channels can eliminated most of the interference from the nonspecific adsorption on the outer surface of MIPs particles, enhancing the anti-interference ability of MIPs sensors greatly. In a modified differential method to correct the difference in specific surface areas between the MIPs and NIPs particle, anti-interference ability of MIPs sensors can be improved further. In the model system for BHb determination using MIPs of GQDs@SiO $/$ CdTe QDs as the sensing material, the interference levels from Lyz, OVA, PA or PC in the modified differential ratiometric fluorescence method were only $-7.1 \% \sim 11 \%$ of the interference levels in the normal ratiometric fluorescence method. The determination of $\mathrm{BHb}$ in real samples on site demonstrated the good potential applicability of the proposed ratiometric fluorescent device, which offered the advantage of portability, sensitivity and reliability for field analysis. Our proposed differential strategy can be used in other MIPs biosensors, such as colorimetric, photoelectrochemical, voltammetric and quartz crystal microbalance methods.

\section{CRediT authorship contribution statement}

Jiangru Wang: Investigation, Writing - original draft. Xiaolan Chen: Investigation, Visualization. Xiaoyan Wang: Methodology, Software. Qi Kang: Data curation, Supervision. Dazhong Shen: Writing - review \& editing, Validation. Lingxin Chen: Conceptualization, Supervision.

\section{Declaration of Competing Interest}

The authors declare that they have no known competing financial interests or personal relationships that could have appeared to influence the work reported in this paper.

\section{Acknowledgments}

The authors gratefully acknowledge financial support of National Natural Science Foundation of China (21874083, 21976209 and 21804010), Natural Science Foundation of Shandong Province of China (ZR2018MB029) and Taishan Scholar Project Special Funding
(No.ts20190962).

\section{Appendix A. Supplementary data}

Supplementary material related to this article can be found, in the online version, at doi:https://doi.org/10.1016/j.snb.2020.128581.

\section{References}

[1] K. Vong, S. Eda, Y. Kadota, I. Nasibullin, T. Wakatake, S. Yokoshima, K. Shirasu, K. Tanaka, An artificial metalloenzyme biosensor can detect ethylene gas in fruits and Arabidopsis leaves, Nat. Commun. 10 (2019) 5746.

[2] S.M. Guo, R. Veneziano, S. Gordonov, L. Li, E. Danielson, K.P. de Arce, D. Park, A.B. Kulesa, E.C. Wamhoff, P.C. Blainey, E.S. Boyden, J.R. Cottrell, M. Bathe, Multiplexed and high-throughput neuronal fluorescence imaging with diffusible probes, Nat. Commun. 10 (2019) 4377.

[3] Y.N. Zhao, X. Li, Y. Yang, S.H. Si, C.Y. Deng, H.Y. Wu, A simple aptasensor for A $\beta_{40}$ oligomers based on tunable mismatched base pairs of dsDNA and graphene oxide, Biosens. Bioelectron. 149 (2020) 111840.

[4] J.J. BelBruno, Molecularly imprinted polymers, Chem. Rev. 119 (2019) 94-119.

[5] M. Yoshikawa, K. Tharpa, S.O. Dima, Molecularly imprinted membranes: past, present, and future, Chem. Rev. 116 (2016) 11500-11528.

[6] L.X. Chen, X.Y. Wang, W.H. Lu, X.Q. Wu, J.H. Li, Molecular imprinting: perspectives and applications, Chem. Soc. Rev. 45 (2016) 2137-2211.

[7] M. Dinc, C. Esen, B. Mizaikoff, Recent advances on coreeshell magnetic molecularly imprinted polymers for biomacromolecules, TrAC Trends Anal. Chem. 114 (2019) 202-217.

[8] L.M. Madikizela, N.T. Tavengwa, H. Tutu, L. Chimuka, Green aspects in molecular imprinting technology: from design to environmental applications, Trends Environ. Anal. Chem. 17 (2018) 14-22.

[9] G. Sharma, B. Kandasubramanian, Molecularly imprinted polymers for selective recognition and extraction of heavy metal ions and toxic dyes, J. Chem. Eng. Data 65 (2020) 396-418.

[10] V. Pichon, N. Delaunay, A. Combès, Sample preparation using molecularly imprinted polymers, Anal. Chem. 92 (2020) 16-33.

[11] Y.R. Cao, T.Y. Feng, J. Xu, C.H. Xue, Recent advances of molecularly imprinted polymer-based sensors in the detection of food safety hazard factors, Biosens. Bioelectron. 141 (2019) 111447.

[12] R.J. Gui, H. Jin, H.J. Guo, Z.H. Wang, Recent advances and future prospects in molecularly imprinted polymers-based electrochemical biosensors, Biosens. Bioelectron. 100 (2018) 56-70.

[13] B. Yang, C. Fu, J.P. Li, G.B. Xu, Frontiers in highly sensitive molecularly imprinted electrochemical sensors: challenges and strategies, TrAC Trends Anal. Chem. 105 (2018) 52-67.

[14] N. Zhang, N. Zhang, Y.R. Xu, Z.L. Li, C.R. Yan, K. Mei, M.L. Ding, S.C. Ding, P. Guan, L.W. Qian, C.B. Du, X.L. Hu, Molecularly imprinted materials for selective biological recognition, Macromol. Rapid Comm. 40 (2019) 1900096.

[15] M. Gast, H. Sobek, B. Mizaikoff, Advances in imprinting strategies for selective virus recognition a review, TrAC Trends Anal. Chem. 114 (2019) 218-232.

[16] J.E. Lofgreen, G.A. Ozin, Controlling morphology and porosity to improve performance of molecularly imprinted sol-gel silica, Chem. Soc. Rev. 43 (2014) 911-933.

[17] A. Bigdeli, F. Ghasemi, S. Abbasi-Moayed, M. Shahrajabian, N. Fahimi- Kashani, S. Jafarinejad, M.A.F. Nejad, M.R. Hormozi-Nezhad, Ratiometric fluorescent nanoprobes for visual detection: design principles and recent advances-a review, Anal. Chim. Acta 1079 (2019) 30-58.

[18] L. Uzun, A.P.F. Turner, Molecularly-imprinted polymer sensors: realising their potential, Biosens. Bioelectron. 76 (2016) 131-144.

[19] A. Roda, E. Michelini, M. Zangheri, M. Di Fusco, D. Calabria, P. Simoni, Smartphone based biosensors: a critical review and perspectives, TrAC Trends Anal. Chem. 79 (2016) (2016) 317-325.

[20] S. Kanchi, M.I. Sabela, P.S. Mdluli, Inamuddin, K. Bisetty, Smartphone based bioanalytical and diagnosis applications: a review, Biosens. Bioelectron. 102 (2018) $136-149$.

[21] S.N. Zulkifli, H.A. Rahim, W.J. Lau, Detection of contaminants in water supply: a review on state-of-the-art monitoring technologies and their applications, Sens. Actuators B 255 (2018) 2657-2689.

[22] D. Capoferri, R. Álvarez-Diduk, M. Del Carlo, D. Compagnone, I.A. Merkoç, Electrochromic molecular imprinting sensor for visual and smartphone-based detections, Anal. Chem. 90 (2018) 5850-5856.

[23] G. Chen, C. Fang, H.H. Chai, Y. Zhou, W.Y. Li, L. Yu, Improved analytical performance of smartphone-based colorimetric analysis by using a power-free imaging box, Sens. Actuators B 281 (2019) 253-261.

[24] K.G. Shah, V. Singh, P.C. Kauffman, K. Abe, P. Yager, Mobile phone ratiometric imaging enables highly sensitive fluorescence lateral flow immunoassays without external optical filters, Anal. Chem. 90 (2018) 6967-6974.

[25] M.C. Rong, Y.C. Liang, D.L. Zhao, B.J. Chen, C. Pan, X.Z. Deng, Y.B. Chen, J. He, A ratiometric fluorescence visual test paper for an anthrax biomarker based on functionalized manganese-doped carbon dots, Sens. Actuators B 265 (2018) 498-505.

[26] J. L.Yang, Z. Li, Q. Jia, Design of dual-emission fluorescence sensor based on Cu nanoclusters with solvent-dependent effects: visual detection of water via a smartphone, Sens. Actuators B 297 (2019) 126807.

[27] A. Shahvar, M. Saraji, D. Shamsaei, Smartphone-based chemiluminescence sensing 
for TLC imaging, Sens. Actuators B 255 (2018) 891-894.

[28] S. Li, J.L. Liu, Z.T. Chen, Y.L. Lu, S.S. Low, L.H. Zhu, C. Cheng, Y. He, Q.M. Chen, B. Su, Q.J. Liu, Electrogenerated chemiluminescence on smartphone with graphene quantum dots nanocomposites for Escherichia coli detection, Sens. Actuators B 297 (2019) 126811.

[29] M. Rezazadeh, S. Seidi, M. Lid, S. Pedersen-Bjergaard, Y. Yamini, The modern role of smartphones in analytical chemistry, TrAC Trends Anal. Chem. 118 (2019) $548-555$.

[30] P.P. Lv, D.D. Xie, Z.H. Zhang, Magnetic carbon dots based molecularly imprinted polymers for fluorescent detection of bovine hemoglobin, Talanta 188 (2018) 145-151.

[31] X.Y. Xie, Q. Hu, R.F. Ke, X.Y. Zhen, Y.S. Bu, S.C. Wang, Facile preparation of photonic and magnetic dual responsive protein imprinted nanomaterial for specific recognition of bovine hemoglobin, Chem. Eng. J. 371 (2019) 130-137.

[32] C. Yang, X.F. Ji, W.Q. Cao, J. Wang, Q. Zhang, T.L. Zhong, Y. Wang, An ultra sensitive and selective impedance sensor based on protein imprinted polymer, Sens. Actuators B 282 (2019) 818-823.

[33] Y.J. Liu, Y.Z. Wang, Q.Z. Dai, Y.G. Zhou, Magnetic deep eutectic solvents molecularly imprinted polymers for the selective recognition and separation of protein, Anal. Chim. Acta 936 (2016) 168-178.

[34] Y.X. Li, Y.T. Chen, L. Huang, B.Y. Lou, G.N. Chen, Creating BHb-imprinted magnetic nanoparticles with multiple binding sites, Analyst 142 (2017) 302-309.

[35] W. Xu, Y.Z. Wang, X.X. Wei, J. Chen, P.L. Xu, R. Ni, J.J. Meng, Y.G. Zhou, Fabrication of magnetic polymers based on deep eutectic solvent for separation of bovine hemoglobin via molecular imprinting technology, Anal. Chim. Acta 1048 (2019) 1-11.

[36] Q. Yang, J.H. Li, X.Y. Wang, H. Xiong, L.X. Chen, Ternary emission of a blue-, green-, and red-based molecular imprinting fluorescence sensor for the multiplexed and visual detection of bovine hemoglobin, Anal. Chem. 91 (2019) 6561-6568.

[37] X.Y. Wang, J.L. Yu, Q. Kang, D.Z. Shen, J.H. Li, L.X. Chen, Molecular imprinting ratiometric fluorescence sensor for highly selective and sensitive detection of phycocyanin, Biosens. Bioelectron. 77 (2016) 624-630.

[38] J. Wang, Q. Xu, W.W. Xia, Y. Shu, D.Q. Jin, Y. Zang, X.Y. Hu, High sensitive visible light photoelectrochemical sensor based on in-situ prepared flexible $\mathrm{Sn}_{3} \mathrm{O}_{4}$ nanosheets and molecularly imprinted polymers, Sens. Actuators B 271 (2018) 215-224.

[39] Y. Gu, Y.N. Wang, X.M. Wu, M.F. Pan, N. Hu, J.P. Wang, S. Wang, Quartz crystal microbalance sensor based on covalent organic framework composite and molecularly imprinted polymer of poly[o-aminothiophenol] with gold nanoparticles for the determination of aflatoxin B1, Sens. Actuators B 291 (2019) 293-297.

[40] R.R. Xing, Y. R.Wen, Y.R. Dong, Y.J. Wang, Q. Zhang, Z. Liu, Dual molecularly imprinted polymer-based plasmonic immunosandwich assay for the specific and sensitive detection of protein biomarkers, Anal. Chem. 91 (2019) 9993-10000.

[41] L.H. Luo, F. Zhang, C.Y. Chen, C.Q. Cai, Visual simultaneous detection of Hepatitis $\mathrm{A}$ and $\mathrm{B}$ viruses based on a multifunctional molecularly imprinted fluorescence sensor, Anal. Chem. 91 (2019) 15748-15756.

[42] J.Y. Yang, W.B. Feng, K.S. Liang, C.Y. Chen, C.Q. Cai, A novel fluorescence molecularly imprinted sensor for Japanese encephalitis virus detection based on metal organic frameworks and passivation-enhanced selectivity, Talanta 212 (2020) 120744.

[43] Y.Q. Dong, J.W. Shao, C.Q. Chen, H. Li, R.X. Wang, Y.W. Chi, X.M. Lin, G.N. Chen, Blue luminescent graphene quantum dots and grapheme oxide prepared by tuning the carbonization degree of citric acid, Carbon 50 (2012) 4738-4743.

[44] F. Ma, M.T. Sun, K. Zhang, S.H. Wang, A ratiometric fluorescence sensor for highly selective and sensitive detection of mercuric ion, Sens. Actuators B 209 (2015) 377-383.

[45] J.L. Yu, X.Y. Wang, Q. Kang, J.H. Li, D.Z. Shen, L.X. Chen, One-pot synthesis of a quantum dot-based molecular imprinting nanosensor for highly selective and sensitive fluorescence detection of 4-nitrophenol in environmental waters, Environ. Sci-Nano 4 (2017) 493-502.

[46] Y.Q. Wang, Y. Liu, X.W. He, W.Y. Li, Y.K. Zhang, Highly sensitive synchronous fluorescence determination of mercury[II] based on the denatured ovalbumin coated CdTe QDs, Talanta 99 (2012) 69-74.

[47] X.Y. Wang, S.M. Yu, W. Liu, L.W. Fu, Y.Q. Wang, J.H. Li, L.X. Chen, Molecular imprinting based hybrid ratiometric fluorescence sensor for the visual determination of bovine hemoglobin, ACS Sens. 3 (2018) 378-385.

[48] G.A. Crosby, J.N. Demas, Measurement of photoluminescence quantum yields, re view, J. Phys. Chem. 75 (1971) 991-1024.

[49] S. Seyedi, P. Parvin, A. Jafargholi, S. Jelvani, M. Shahabi, M. Shahbazi, P. Mohammadimatin, A. Moafi, Fluorescence properties of phycocyanin and phycocyanin-human serum albumin complex, Spectrochim. Acta A 239 (2020) 118468.

[50] D.Y. Li, X.W. He, Y. Chen, W.Y. Li, Y.K. Zhang, Novel hybrid structure silica/CdTe/ molecularly imprinted polymer: synthesis, specific recognition, and quantitative fluorescence detection of bovine hemoglobin, ACS Appl. Mater. Interfaces 5 (2013) 12609-12616.

[51] T. Guo, Q.L. Deng, G.Z. Fang, D.H. Gu, Y.K. Yang, S. Wang, Upconversion fluorescence metal-organic frameworks thermosensitive imprinted polymer for enrichment and sensing protein, Biosens. Bioelectron. 79 (2016) 341-346.
[52] L. Tan, C.C. Kang, S.Y. Xu, Y.W. Tang, Selective room temperature phosphorescence sensing of target protein using Mn-doped ZnS QDs-embedded molecularly imprinted polymer, Biosens. Bioelectron. 48 (2013) 216-223.

[53] H.Z. Lu, S.F. Xu, Functional monomer-template-QDs sandwich structure for mesoporous structured bovine hemoglobin imprinted ratiometric fluorescence sensor, Talanta 165 (2017) 482-488.

[54] M.F. Jia, Z. Zhang, J.H. Li, H.J. Shao, L.X. Chen, X.B. Yang, A molecular imprinting fluorescence sensor based on quantum dots and a mesoporous structure for selective and sensitive detection of 2,4-dichlorophenoxyacetic acid, Sens. Actuators B 252 (2017) 934-943.

[55] X.Y. Wang, J.L. Yu, J.H. Li, Q. Kang, D.Z. Shen, L.X. Chen, Quantum dots based imprinting fluorescent nanosensor for the selective and sensitive detection of phy cocyanin: a general imprinting strategy toward proteins, Sens. Actuators, B 255 (2018) 268-274

[56] H.H. Wang, X.J. Chen, W.T. Li, W.H. Zhou, X.C. Guo, W.Y. Kang, D.X. Kou, Z.J. Zhou, Y.N. Meng, Q.W. Tian, S.X. Wu, ZnO nanotubes supported molecularly imprinted polymers arrays as sensing materials for electrochemical detection of dopamine, Talanta 176 (2018) 573-581.

[57] X.L. Sun, Y.N. Jian, H. Wang, S.G. Ge, M. Yan, J.H. Yu, Ultrasensitive microfluidic paper-based electrochemical biosensor based on molecularly imprinted film and boronate affinity sandwich assay for glycoprotein detection, ACS Appl. Mater. Interfaces 11 (2019) 16198-16206.

[58] T. Ye, W.X. Yin, N.X. Zhu, M. Yuan, H. Cao, J.S. Yu, Z.Q. Gou, X. Wang, H.S. Zhu, A. Reyihanguli, F. Xu, Colorimetric detection of pyrethroid metabolite by using surface molecularly imprinted polymer, Sens. Actuators B 254 (2018) 417-423.

[59] S.Y. Huang, M.L. Guo, J.A. Tan, Y.Y. Geng, J.Y. Wu, Y.W. Tang, C.C. Su, C.C. Lin, Y. Liang, Novel fluorescence sensor based on all-inorganic perovskite quantum dots coated with molecularly imprinted polymers for highly selective and sensitive detection of omethoate, ACS Appl. Mater. Interfaces 10 (2018) 39056-39063.

[60] Q. Yang, J.H. Li, X.Y. Wang, H.L. Peng, H. Xiong, L.X. Chen, Dual-emission colorcontrollable nanoparticle based molecular imprinting ratiometric fluorescence sensor for the visual detection of Brilliant Blue, Sens. Actuators B 284 (2019) 428-436.

[61] E. Petryayeva, W.R. Algar, Multiplexed homogeneous assays of proteolytic activity using a smartphone and quantum dots, Anal. Chem. 86 (2014) 3195-3202.

[62] H.H. Wang, Y. Sun, H.J. Li, W. W.Yue, Q. Kang, D.Z. Shen, A smartphone-based ratiometric resonance light scattering device for field analysis of $\mathrm{Pb}^{2+}$ in river water samples and immunoassay of alpha fetoprotein using PbS nanoparticles as signal tag, Sens. Actuators- B 271 (2018) 358-366.

Jiangru Wang was received her BS degree in 2016 in chemistry and MSc degrees in 2019 in analytical chemistry from Shandong Normal University. She is studying for her doctoral degree in analytical chemistry at the same university. Her current research interest is molecularly imprinted polymers based chemical sensors for biological analysis.

Xiaolan Chen was received her BS degree in 2018 in chemistry from College of Materials and Chemical Engineering, Hezhou University. She is studying for her master degree in analytical chemistry at Shandong Normal University. Her current research interest is smartphone based analytical devices.

Xiaoyan Wang received her $\mathrm{PhD}$ in Shandong Normal University, Joint-Educated at Yantai Institute of Coastal Zone Research, Chinese Academy of Sciences, in 2017. Her current research interests focus on the preparation \& application of molecularly imprinted polymers in chromatographic separation and chemical sensors for analysis of typical pollutants.

Qi Kang is a professor in School of Chemistry, Chemical Engineering and Material Science, Shandong Normal University. She received her MSc and PhD degrees in analytical chemistry from Shandong University. Her research interests include chemical sen-

Dazhong Shen is a professor in School of Chemistry, Chemical Engineering and Material Science, Shandong Normal University. He received his MSc and PhD degrees in analytical chemistry from Hunan University. His research interests include chemical sensors and biosensors.

Lingxin Chen has been a professor at Yantai Institute of Coastal Zone Research, Chinese Academy of Sciences, since 2009. He obtained his Ph.D. in analytical chemistry at Dalian Institute of Chemical Physics, Chinese Academy of Sciences, in 2003. During 2004-2009, he worked at Department of Chemistry, Tsinghua University, and Department of Applied Chemistry, Hanyang University, respectively. His research interests include the studies of novel properties of materials such as functionalized nanoparticles \& functional probe molecules for developing nanoscale biochemical analysis methods and molecular imprinting-based sample pretreatment technology. He has published 3 academic books, 200 research papers and topical reviews. 